\title{
A GENERALIZED TWO-POINT BOUNDARY VALUE PROBLEM
}

\section{J. W. BEBERNES ${ }^{1}$ AND ROBERT GAINES ${ }^{2}$}

1. Introduction. The purpose of this paper is to prove there exists a unique solution of the two-point boundary value problem

(1.1) $x^{\prime \prime}=f\left(t, x, x^{\prime}\right)$,

(1.2) $a_{0} x(a)-a_{1} x^{\prime}(a)=\alpha$,

(1.3) $b_{0} x(b)+b_{1} x^{\prime}(b)=\beta$

assuming that:

(i) $f\left(t, x, x^{\prime}\right)$ is a continuous real valued function defined on $S \equiv\left\{\left(t, x, x^{\prime}\right)|a \leqq t \leqq b| x,|+| x^{\prime} \mid<\infty\right\}$,

(ii) $f\left(t, x, x^{\prime}\right)$ is nondecreasing on $S$ with respect to $x$,

(iii) $\left|f\left(t, x, x_{1}^{\prime}\right)-f\left(t, x, x_{2}^{\prime}\right)\right| \leqq M\left|x_{1}^{\prime}-x_{2}^{\prime}\right|$ on $S$,

and in addition assuming that $a_{0}, a_{1}, b_{0}, b_{1} \geqq 0, a_{0}+b_{0}>0, a_{0}+a_{1}>0$, and $b_{0}+b_{1}>0$.

By boundary value problem (BVP) (1.1), we shall mean equation (1.1) together with boundary conditions (1.2) and (1.3). BVP(1.1) was investigated in [5]. In this paper, the assumptions on $f\left(t, x, x^{\prime}\right)$ are weakened considerably and a much simpler proof of the existence of a unique solution to $\mathrm{BVP}(1.1)$ is given.

The main result of this note is Theorem 3.1. The proof makes use of the existence and uniqueness theory developed using the subfunction approach in [1] and [2]. In particular, under conditions (i), (ii), and (iii), the two-point boundary value problem: $x^{\prime \prime}=f\left(t, x, x^{\prime}\right)$, $x(a)=\gamma, x(b)=\delta$, has a unique solution $u(t, \gamma, \delta)$ which depends continuously on the boundary data $(\gamma, \delta)$.

2. Preliminary results. In this section, we prove a sequence of lemmas.

Lemma 2.1. Let $\phi(s)$ be a positive continuous function such that

$$
\int^{\infty} \frac{s d s}{\phi(s)}=\infty .
$$

Given $R>0$, there exists $M_{R}>0$ such that if $x(t) \in C^{2}[a, b]$, satisfies $|x(t)| \leqq R$ and $\left|x^{\prime \prime}(t)\right| \leqq \phi\left(\left|x^{\prime}(t)\right|\right)$ on $[a, b]$, then $\left|x^{\prime}(t)\right| \leqq M_{R}$. Moreover, $M_{R} \rightarrow 0$ as $R \rightarrow 0$.

Proof. Given $R>0$, by (2.1) there exists $M_{R}>0$ such that

Received by the editors March 16, 1967.

1 Partially supported under Grant NSF-GP-5965.

2 Supported by a NASA Traineeship. 


$$
\int_{2 R /(b-a)}^{M_{R}} \frac{s d s}{\phi(s)}=2 R
$$

Then $\left|x^{\prime}(t)\right| \leqq M_{R}$ follows (Hartman [3, pp. 428-429]). From (2.2), we see that if $R \rightarrow 0$, then $M_{R} \rightarrow 0$.

Lemma 2.2. If $f\left(t, x, x^{\prime}\right)$ satisfies (i), (ii), (iii), then the boundary value problem

$$
x^{\prime \prime}=f\left(t, x, x^{\prime}\right) \quad x(c)=\gamma, \quad x(d)=\delta
$$

has a unique solution $u(t, \gamma, \delta)$ for any $a \leqq c \leqq d \leqq b$.

Proof. This is a corollary to [1, Theorem 6.2, p. 1064].

Lemma 2.3. If $f\left(t, x, x^{\prime}\right)$ satisfies (i), (ii), (iii), then $u(t, \bar{\gamma}, \bar{\delta})$ $\rightarrow u(t, \gamma, \delta)$ and $u^{\prime}(t, \bar{\gamma}, \bar{\delta}) \rightarrow u^{\prime}(t, \gamma, \delta)$ uniformly on $[c, d]$ as $|\gamma-\bar{\gamma}|$ $+|\delta-\bar{\delta}| \rightarrow 0$.

Proof. Given $\epsilon>0$, by Jackson and Fountain [2, Theoren 9, p. 1262], $u(t, \gamma, \delta)+\epsilon$ is a superfunction and $u(t, \gamma, \delta)-\epsilon$ is a subfunction relative to solutions of $x^{\prime \prime}=f\left(t, x, x^{\prime}\right)$. Hence, if $|\bar{\delta}-\delta|+|\bar{\gamma}-\gamma|$ $<\epsilon$, then $u(t, \gamma, \delta)-\epsilon \leqq u(t, \bar{\gamma}, \bar{\delta}) \leqq u(t, \gamma, \delta)+\epsilon$ for all $t \in[c, d]$; and $u(t, \bar{\gamma}, \bar{\delta}) \rightarrow u(t, \gamma, \delta)$ uniformly on $[c, d]$ as $|\bar{\delta}-\delta|+|\bar{\gamma}-\gamma| \rightarrow 0$.

We have left to prove that $u^{\prime}(t, \bar{\gamma}, \bar{\delta}) \rightarrow u^{\prime}(t, \gamma, \delta)$ as $|\bar{\gamma}-\gamma|$ $+|\bar{\delta}-\delta| \rightarrow 0$.

Define $K \equiv\{(t, y) \mid c \leqq t \leqq d, u(t, \gamma-1, \delta-1) \leqq y \leqq u(t, \gamma+1, \delta+1)\}$ and $\phi(s) \equiv M s+\max _{K}|f(t, y, 0)|$. Note that $\int^{\infty}(s d s / \phi(s))=\infty$. For $|\gamma-\bar{\gamma}| \leqq 1$ and $|\delta-\bar{\delta}| \leqq 1$, we have

$$
\begin{aligned}
\left|u^{\prime \prime}(t, \bar{\gamma}, \delta)\right| & =\left|f\left(t, u(t, \bar{\gamma}, \bar{\delta}), u^{\prime}(t, \bar{\gamma}, \bar{\delta})\right)\right| \\
& \leqq M\left|u^{\prime}(t, \bar{\gamma}, \bar{\delta})\right|+|f(t, u(t, \bar{\gamma}, \bar{\delta}), 0)| \\
& \leqq \phi\left(\left|u^{\prime}(t, \bar{\gamma}, \bar{\delta})\right|\right)
\end{aligned}
$$

and

$$
\begin{aligned}
|u(t, \bar{\gamma}, \bar{\delta})| & \leqq B \\
& \equiv \max _{[a, b]}\{\max [|u(t, \gamma-1, \delta-1)|,|u(t, \gamma+1, \delta+1)|]\} .
\end{aligned}
$$

Hence, by Lemma 2.1 , there exists $N_{B}>0$ such that $\left|u^{\prime}(t, \bar{\gamma}, \bar{\delta})\right| \leqq N_{B}$ for $|\gamma-\bar{\gamma}| \leqq 1$ and $|\delta-\bar{\delta}| \leqq 1$.

Define $\phi_{1}(s) \equiv 2 \max \left|f\left(t, y, y^{\prime}\right)\right|$ over $\left\{\left(t, y, y^{\prime}\right)|(t, y) \in K,| y^{\prime} \mid \leqq N_{B}\right\}$ and $R \equiv \max |u(t, \bar{\gamma}, \bar{\delta})-u(t, \gamma, \delta)|$ on $[c, d]$. Note that $\int^{\infty}\left(s d s / \phi_{1}(s)\right)$ $=\infty$. For $|\gamma-\bar{\gamma}| \leqq 1$ and $|\delta-\bar{\delta}| \leqq 1$, we have 


$$
\begin{aligned}
\left|u^{\prime \prime}(t, \bar{\gamma}, \bar{\delta})-u^{\prime \prime}(t, \gamma, \delta)\right| \leqq & \left|f\left(t, u(t, \bar{\gamma}, \bar{\delta}), u^{\prime}(t, \bar{\gamma}, \bar{\delta})\right)\right| \\
& +\left|f\left(t, u(t, \gamma, \delta), u^{\prime}(t, \gamma, \delta)\right)\right| \\
\leqq & \phi_{1}\left(\left|u^{\prime}(t, \bar{\gamma}, \bar{\delta})-u^{\prime}(t, \gamma, \delta)\right|\right) .
\end{aligned}
$$

By Lemma 2.1, $\left|u^{\prime}(t, \bar{\gamma}, \bar{\delta})-u^{\prime}(t, \gamma, \delta)\right| \leqq M_{R}$ where $M_{R} \rightarrow 0$ as $R \rightarrow 0$. Since $R \rightarrow 0$ as $|\gamma-\bar{\gamma}|+|\delta-\bar{\delta}| \rightarrow 0$, we conclude that $u^{\prime}(t, \bar{\gamma}, \bar{\delta})$ $\rightarrow u^{\prime}(t, \gamma, \delta)$ uniformly on $[c, d]$ as $|\gamma-\bar{\gamma}|+|\delta-\bar{\delta}| \rightarrow 0$.

Lemma 2.4. If $f\left(t, x, x^{\prime}\right)$ satisfies (i), (ii), and (iii), $\gamma_{2} \geqq \gamma_{1}$ and $\delta_{2} \geqq \delta_{1}$, then $z(t) \equiv u\left(t, \gamma_{2}, \delta_{2}\right)-u\left(t, \gamma_{1}, \delta_{1}\right)$ is a subfunction with respect to solutions of

$$
u^{\prime \prime}=-M\left|u^{\prime}\right|
$$

on $[c, d]$. If $\delta_{2}-\delta_{1} \geqq \gamma_{2}-\gamma_{1}$, then

$$
\begin{aligned}
& 0 \leqq z(t) \leqq\left(\gamma_{2}-\gamma_{1}\right)+\left[\left(\delta_{2}-\delta_{1}\right)-\left(\gamma_{2}-\gamma_{1}\right)\right] \\
& \cdot\left[\int_{c}^{t} \exp (-M(s-c)) d s\right] /\left[\int_{c}^{d} \exp (-M(s-c)) d s\right] \\
& \equiv \omega_{1}(t) .
\end{aligned}
$$

If $\delta_{2}-\delta_{1} \leqq \gamma_{2}-\gamma_{1}$, then

$$
\begin{aligned}
0 \leqq z(t) \leqq\left(\gamma_{2}-\gamma_{1}\right)+\left[\left(\delta_{2}-\delta_{1}\right)-\left(\gamma_{2}-\gamma_{1}\right)\right] \\
\cdot\left[\int_{c}^{t} \exp (M(s-c)) d s\right] /\left[\int_{c}^{d} \exp (M(s-c)) d s\right] \\
\equiv \omega_{2}(t) .
\end{aligned}
$$

Proof. By the uniqueness of solutions to the two-point boundary value problem in Lemma 2.2 , we must have $u\left(t, \gamma_{2}, \delta_{2}\right) \geqq u\left(t, \gamma_{1}, \delta_{1}\right)$. By properties (ii) and (iii),

$$
\begin{aligned}
z^{\prime \prime}(t) & =f\left(t, u\left(t, \gamma_{2}, \delta_{2}\right), u^{\prime}\left(t, \gamma_{2}, \delta_{2}\right)\right)-f\left(t, u\left(t, \gamma_{1}, \delta_{1}\right), u^{\prime}\left(t, \gamma_{1}, \delta_{1}\right)\right) \\
& \geqq f\left(t, u\left(t, \gamma_{1}, \delta_{1}\right), u^{\prime}\left(t, \gamma_{2}, \delta_{2}\right)\right)-f\left(t, u\left(t, \gamma_{1}, \delta_{1}\right), u^{\prime}\left(t, \gamma_{1}, \delta_{1}\right)\right) \\
& \geqq-M\left|u^{\prime}\left(t, \gamma_{2}, \delta_{2}\right)-u^{\prime}\left(t, \gamma_{1}, \delta_{1}\right)\right|=-M\left|z^{\prime}(t)\right| .
\end{aligned}
$$

By Jackson and Fountain [2, Theorem 8, p. 1261], this inequality implies that $z(t)$ is a subfunction with respect to $(2.4)$ on $[c, d]$.

If $\delta_{2}-\delta_{1} \geqq \gamma_{2}-\gamma_{1}$, then $\omega_{1}(t)$ is a solution to (2.4) satisfying $\omega_{1}(c)$ $=z(c)$ and $\omega_{1}(d)=z(d)$. Similarly, if $\gamma_{2}-\gamma_{1} \leqq \delta_{2}-\delta_{1}$, then $\omega_{2}(t)$ is such a solution. Then inequalities (2.5) and (2.6) follow from the definition of subfunction (see Jackson and Fountain [2, p. 1254]).

Lemma 2.5. If $f\left(t, x, x^{\prime}\right)$ satisfies (i), (ii), and (iii) and if $b_{0}, b_{1} \geqq 0$ 
and $b_{0}+b_{1}>0$, then the boundary value problem

$$
x^{\prime \prime}=f\left(t, x, x^{\prime}\right) \quad x(a)=s, \quad b_{0} x(b)+b_{1} x^{\prime}(b)=\beta
$$

has a unique solution $u(t, s, \delta(s))$ on $[a, b]$ where $u(b, s, \delta(s))=\delta(s)$.

Proof. By Lemma 2.2, the problem

$$
x^{\prime \prime}=f\left(t, x, x^{\prime}\right) \quad x(a)=s, \quad x(b)=\delta
$$

has a unique solution $u(t, s, \delta)$ on $[a, b]$.

Define $F_{s}(\delta) \equiv b_{0} \delta+b_{1} u^{\prime}(b, s, \delta)$. It is sufficient to show that there exists a unique $\delta(s)$ such that $F_{s}(\delta(s))=\beta$.

From Lemma 2.3 we have that $u^{\prime}(b, s, \delta)$ is a continuous function of $\delta$ on $(-\infty,+\infty)$ for fixed $s$. Hence, $F_{s}(\delta)$ is continuous on $(-\infty$, $\infty)$. Suppose $\delta_{2} \geqq \delta_{1}$. We have

$$
F_{s}\left(\delta_{2}\right)-F_{s}\left(\delta_{1}\right)=b_{0}\left(\delta_{2}-\delta_{1}\right)+b_{1}\left(u^{\prime}\left(b, s, \delta_{2}\right)-u^{\prime}\left(b, s, \delta_{1}\right)\right) .
$$

Applying Lemma 2.4 (with $\gamma_{1}=\gamma_{2}=s$ ), since $z(b)=u\left(b, s, \delta_{2}\right.$ ) $-u\left(b, s, \delta_{1}\right)=\omega_{1}(b)$, we have

$$
\begin{aligned}
z^{\prime}(b) & =u^{\prime}\left(b, s, \delta_{2}\right)-u^{\prime}\left(b, s, \delta_{1}\right) \geqq \omega_{1}^{\prime}(b) \\
& =\left(\delta_{2}-\delta_{1}\right) \exp [-M(b-a)] / \int_{a}^{b} \exp [-M(s-a)] d s .
\end{aligned}
$$

Define $P_{1} \equiv b_{0}+b_{1} \exp [-M(b-a)] / \int_{a}^{b} \exp [-M(s-a)] d s>0$. Then,

$$
F_{s}\left(\delta_{2}\right)-F_{s}\left(\delta_{1}\right) \geqq P_{1}\left(\delta_{2}-\delta_{1}\right) .
$$

This inequality implies that $F_{\boldsymbol{s}}(\delta)$ is strictly increasing and has $(-\infty,+\infty)$ for its range. Hence, there exists a unique $\delta(s)$ such that $F_{s}(\delta(s))=\beta$, and $u(t, s, \delta(s))$ is the unique solution to (2.7).

Lemma 2.6. If $f\left(t, x, x^{\prime}\right)$ satisfies (i), (ii), and (iii) and if $b_{0}, b_{1} \geqq 0$ and $b_{0}+b_{1}>0$, then $u(t, \bar{s}, \delta(\bar{s})) \rightarrow u(t, s, \delta(s))$ and $u^{\prime}(t, \bar{s}, \delta(\bar{s}))$ $\rightarrow u^{\prime}(t, s, \delta(s))$ uniformly on $[a, b]$ as $\bar{s} \rightarrow s$. Moreover, if $s_{2} \geqq s_{1}$, then

$$
0 \leqq u\left(t, s_{2}, \delta\left(s_{2}\right)\right)-u\left(t, s_{1}, \delta\left(s_{1}\right)\right) \leqq s_{2}-s_{1} \text {. }
$$

Proof. We first establish that (2.9) holds at $b$, i.e. $0 \leqq \delta\left(s_{2}\right)$ $-\delta\left(s_{1}\right) \leqq s_{2}-s_{1}$.

If $b_{1}=0$, then $\delta\left(s_{2}\right)-\delta\left(s_{1}\right)=\beta / b_{0}-\beta / b_{0}=0$.

Suppose $b_{1}>0$. Since $u(t, s, \delta(s))$ is a solution to (2.7) for every $s$,

$$
u^{\prime}\left(b, s_{2}, \delta\left(s_{2}\right)\right)-u^{\prime}\left(b, s_{1}, \delta\left(s_{1}\right)\right)=-\left(b_{0} / b_{1}\right)\left(\delta\left(s_{2}\right)-\delta\left(s_{1}\right)\right)
$$

Suppose $\delta\left(s_{2}\right)-\delta\left(s_{1}\right)<0$. Then (2.10) implies that

$$
u^{\prime}\left(b, s_{2}, \delta\left(s_{2}\right)\right)-u^{\prime}\left(b, s_{1}, \delta\left(s_{1}\right)\right) \geqq 0 .
$$


Define $t_{0} \equiv \sup \left\{t \mid t \in[a, b]\right.$ and $\left.u\left(t, s_{2}, \delta\left(s_{2}\right)\right)=u\left(t, s_{1}, \delta\left(s_{1}\right)\right)\right\}$. Note that $t_{0} \in[a, b)$. Applying Lemma 2.4 (with $\gamma_{1}=\gamma_{2}=u\left(t_{0}, s_{2}, \delta\left(s_{2}\right)\right.$ ), $\left.\delta_{2}=\delta\left(s_{1}\right), \delta_{1}=\delta\left(s_{2}\right),[c, d]=\left[t_{0}, b\right]\right)$, since $\delta\left(s_{1}\right)>\delta\left(s_{2}\right)$ and $z(b)=\delta\left(s_{1}\right)$ $-\delta\left(s_{2}\right)=\omega_{1}(b)$, we obtain

$$
\begin{aligned}
z^{\prime}(b)= & u^{\prime}\left(b, s_{1}, \delta\left(s_{1}\right)\right)-u^{\prime}\left(b, s_{2}, \delta\left(s_{2}\right)\right) \geqq \omega_{1}^{\prime}(b) \\
= & \left(\delta\left(s_{1}\right)-\delta\left(s_{2}\right)\right) \\
& \cdot \exp \left(-M\left(b-t_{0}\right)\right) / \int_{t_{0}}^{b} \exp (-M(s-a)) d s>0 .
\end{aligned}
$$

But (2.11) and (2.12) yield a contradiction. Hence, $\delta\left(s_{2}\right)-\delta\left(s_{1}\right) \geqq 0$. Suppose $\delta\left(s_{2}\right)-\delta\left(s_{1}\right)>s_{2}-s_{1}$. Applying Lemma 2.4 (with $\gamma_{1}=s_{1}$, $\gamma_{2}=s_{2}, \delta_{1}=\delta\left(s_{1}\right)$, and $\left.\delta_{2}=\delta\left(s_{2}\right)\right)$, since $z(b)=\delta\left(s_{2}\right)-\delta\left(s_{1}\right)=\omega_{1}(b)$, we obtain

$$
\boldsymbol{z}^{\prime}(b)=u^{\prime}\left(b, s_{2}, \delta\left(s_{2}\right)\right)-u^{\prime}\left(b, s_{1}, \delta\left(s_{1}\right)\right)
$$

$$
\geqq \omega_{1}^{\prime}(b)=\frac{\left[\left(\delta\left(s_{2}\right)-\delta\left(s_{1}\right)\right)-\left(s_{2}-s_{1}\right)\right] \exp (-M(b-a))}{\int_{a}^{b} \exp (-M(s-a)) d s}>0 .
$$

But, since $b_{0} \geqq 0$ and $b_{1}>0$, this contradicts (2.10). Hence $\delta\left(s_{2}\right)-\delta\left(s_{1}\right)$ $\leqq s_{2}-s_{1}$.

Now (2.6) of Lemma 2.4 yields (2.9) since $0 \leqq z(t)=u\left(t, s_{2}, \delta\left(s_{2}\right)\right)$ $-u\left(t, s_{1}, \delta\left(s_{1}\right)\right) \leqq \omega_{2}(t) \leqq\left(s_{2}-s_{1}\right)$. Moreover, $\left|\delta\left(s_{2}\right)-\delta\left(s_{1}\right)\right| \leqq\left|s_{2}-s_{1}\right|$, i.e. $\delta(s)$ is continuous. From Lemma 2.3 we conclude that $u(t, \bar{s}, \delta(\bar{s}))$ $\rightarrow u(t, s, \delta(s))$ and $u^{\prime}(t, \bar{s}, \delta(\bar{s})) \rightarrow u^{\prime}(t, s, \delta(s))$ uniformly on $[a, b]$ as $\bar{s} \rightarrow s$.

Lemma 2.7. If $f\left(t, x, x^{\prime}\right)$ satisfies (i), (ii), (iii) and if $a_{0}, a_{1} \geqq 0$ and $a_{0}+a_{1}>0$, then the boundary value problem

$$
x^{\prime \prime}=f\left(t, x, x^{\prime}\right), \quad x(b)=s, \quad a_{0} x(a)-a_{1} x^{\prime}(a)=\alpha
$$

has a unique solution $u(t, \gamma(s), s)$ on $[a, b]$ where $u(a, \gamma(s), s)=\gamma(s)$. $A s \bar{s} \rightarrow s, u(t, \gamma(\bar{s}), \bar{s}) \rightarrow u(t, \gamma(s), s)$ and $u^{\prime}(t, \gamma(\bar{s}), \bar{s}) \rightarrow u^{\prime}(t, \gamma(s), s)$ uniformly on $[a, b]$. Moreover, if $s_{2} \geqq s_{1}$, then

$$
0 \leqq u\left(t, \gamma\left(s_{2}\right), s_{2}\right)-u\left(t, \gamma\left(s_{1}\right), s_{1}\right) \leqq s_{2}-s_{1} .
$$

Proof. Similar to Lemmas 2.5 and 2.6.

3. Main result. With the aid of the preceding lemmas we can now prove the following which is a generalization of Keller's Theorem [4, p. 728].

Theorem 3.1. If $f\left(t, x, x^{\prime}\right)$ satisfies (i), (ii), and (iii) and if 


$$
\begin{aligned}
a_{0}, a_{1}, b_{0}, b_{1} & \geqq 0, \\
a_{0}+b_{0} & >0, \quad a_{0}+a_{1}>0, \quad b_{0}+b_{1}>0,
\end{aligned}
$$

then the boundary value problem (1.1) has a unique solution $u(t)$ for any $\alpha$ and $\beta$.

Proof. By Lemma 2.5, the problem (2.7) has a unique solution $u(t, s, \delta(s))$ for each $s$. Define $G(s) \equiv a_{0} s-a_{1} u^{\prime}(a, s, \delta(s))$. It is sufficient to show there exists a unique $s$ such that $G(s)=\alpha$. Assume $a_{0}>0$.

By Lemma 2.6, $u^{\prime}(a, s, \delta(s))$ is a continuous function of $s$ on $(-\infty$, $\infty)$. Hence, $G(s)$ is continuous on $(-\infty, \infty)$.

Suppose $s_{2} \geqq s_{1}$. We have

(3.3) $G\left(s_{2}\right)-G\left(s_{1}\right)=a_{0}\left(s_{2}-s_{1}\right)-a_{1}\left(u^{\prime}\left(a, s_{2}, \delta\left(s_{2}\right)\right)-u^{\prime}\left(a, s_{1}, \delta\left(s_{1}\right)\right)\right)$.

By Lemma $2.6,0 \leqq u\left(t, s_{2}, \delta\left(s_{2}\right)\right)-u\left(t, s_{1}, \delta\left(s_{1}\right)\right) \leqq s_{2}-s_{1}$. Moreover, $u\left(a, s_{2}, \delta\left(s_{2}\right)\right)-u\left(a, s_{1}, \quad \delta\left(s_{1}\right)\right)=s_{2}-s_{1}$. Hence, $u^{\prime}\left(a, s_{2}, \delta\left(s_{2}\right)\right)$ $-u^{\prime}\left(a, s_{1}, \delta\left(s_{1}\right)\right) \leqq 0$. We may conclude from (3.3) that

$$
G\left(s_{2}\right)-G\left(s_{1}\right) \geqq a_{0}\left(s_{2}-s_{1}\right) .
$$

This inequality implies that $G(s)$ is strictly increasing and has $(-\infty,+\infty)$ as its range. Hence, there exists a unique $s$ such that $G(s)=\alpha$. Thus, $\operatorname{BVP}(1.1)$ has a unique solution.

If $a_{0}=0$, then we must have $b_{0}>0$, and the proof may be carried out as above by reversing the roles of $a, b$ and using Lemma 2.7.

\section{REFERENCES}

1. J. W. Bebernes, A subfunction approach to a boundary value problem for ordinary differential equations, Pacific J. Math. 13 (1963), 1053-1066.

2. L. Fountain and L. Jackson, A generalized solution of the boundary value problem for $y^{\prime \prime}=f\left(x, y, y^{\prime}\right)$, Pacific J. Math. 12 (1962), 1251-1272.

3. P. Hartman, Ordinary differential equations, Wiley, New York, 1964.

4. H. B. Keller, Existence theory for two-point boundary value problems, Bull. Amer. Math. Soc. 72 (1966), 728-731.

5. J. W. Bebernes and Robert Gaines, Dependence on boundary data and a generalized boundary value problem, J. Differential Equations (to appear).

UNiversity OF COLORADO AND

Colorado State University 